\title{
The Low-Dose (7.5 mg/day) Pioglitazone Therapy
}

\author{
Hidekatsu Yanai $^{\mathrm{a}, \mathrm{b}}$, Hiroki Adachi ${ }^{\mathrm{a}}$
}

\begin{abstract}
Pioglitazone is one of thiazolidinedione derivatives, which stimulates nuclear peroxisome proliferator-activated receptor gamma and improves glucose and lipid metabolism and insulin sensitivity. A recent systematic review and meta-analysis showed that pioglitazone therapy was associated with a lower risk of major adverse cardiovascular events in patients with pre-diabetes and diabetes. Further, in a cohort study of patients with type 2 diabetes, pioglitazone therapy was associated with a statistically significant decrease in the risk of all-cause mortality. Despite these beneficial effects, the metaanalysis showed that pioglitazone therapy had higher risks of heart failure, bone fractures, edema and weight gain. To find out the efficacy and safety of the low-dose $(7.5 \mathrm{mg} /$ day $)$ pioglitazone therapy, we reviewed the dose-response of pioglitazone on favorable effects and adverse effects due to pioglitazone, by searching the reports on effects of daily dose of $7.5 \mathrm{mg}$ and/or $15 \mathrm{mg}$ and/or $30 \mathrm{mg}$ of pioglitazone. The low-dose pioglitazone therapy may show the same degree of improvements in glucose and lipid metabolism, fatty liver, insulin resistance, and adiponectin as the standard- and high-dose pioglitazone therapy. Furthermore, the low-dose pioglitazone therapy may also show less adverse effects on weight gain, edema and heart failure as compared with the standard- and high-dose pioglitazone therapy.
\end{abstract}

Keywords: Body weight; Hear failure; Lipid metabolism; Liver function; Pioglitazone

\section{Introduction}

Pioglitazone is one of thiazolidinedione derivatives, which stimulates nuclear peroxisome proliferator-activated receptor gamma (PPAR $\gamma)$ and improves insulin sensitivity by acting on adipose tissue, muscle and liver [1,2]. Pioglitazone improves

Manuscript submitted July 29, 2017, accepted August 14, 2017

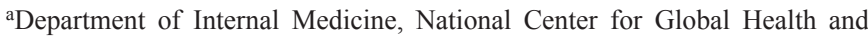
Medicine Kohnodai Hospital, Chiba, Japan

${ }^{\mathrm{b}}$ Corresponding Author: Hidekatsu Yanai, Department of Internal Medicine, National Center for Global Health and Medicine Kohnodai Hospital, 1-7-1 Kohnodai, Ichikawa, Chiba 272-8516, Japan.

Email: dyanai@hospk.ncgm.go.jp

doi: https://doi.org/10.14740/jocmr3144w serum lipids in addition to glucose-lowering [3]. A recent systematic review and meta-analysis showed that pioglitazone therapy was associated with a lower risk of major adverse cardiovascular events (MACEs) in patients with pre-diabetes or insulin resistance (-23\%), and diabetes (-17\%) [4]. Another meta-analysis demonstrated that pioglitazone therapy in stroke patients with insulin resistance, pre-diabetes, and diabetes was associated with lower risk of recurrent stroke (-32\%) and future major vascular events (-25\%) [5]. Further, in a European multi-database cohort study of patients with type 2 diabetes, pioglitazone therapy was associated with a statistically significant decrease in the risk of all-cause mortality [6]. Despite these beneficial effects, pioglitazone therapy had higher risks of heart failure $(+32 \%)$, bone fractures $(+52 \%)$, edema $(+63 \%)$ and weight gain $(+60 \%)$ in the meta-analysis [4].

We previously reported that the low-dose $(7.5 \mathrm{mg} /$ day $)$ pioglitazone therapy is beneficial to the improvement in metabolic parameters without weight gain and an increase of risk for heart failure [7]. To find out the efficacy and safety of the low-dose pioglitazone therapy, we reviewed the dose-response of pioglitazone on favorable effects and adverse effects due to pioglitazone, by searching the reports on effects of daily dose of $7.5 \mathrm{mg}$ and/or $15 \mathrm{mg}$ and/or $30 \mathrm{mg}$ of pioglitazone.

\section{Effects of Low-, Standard- and High-Dose Piogl- itazone Therapy on Efficacy and Safety of Piogl- itazone}

Effects of low- (7.5 mg/day), standard- $(15 \mathrm{mg} /$ day $)$ and highdose $(30 \mathrm{mg} /$ day) pioglitazone therapy on efficacy- and safetyrelated parameters due to pioglitazone were shown in Table 1 [7-10].

In the study by Rajagopalan et al, all three groups showed a significant reduction in $\mathrm{HbAlc}$, fasting plasma glucose (FPG) and postprandial plasma glucose (PPG) [8]. However, there was no significant difference in $\mathrm{HbAlc}$ reduction among three groups. Dose dependency could not be obtained for reduction of HbA1c $(\mathrm{r}=0.09 ; \mathrm{P}=0.42)$, FPG $(\mathrm{r}=0.114 ; \mathrm{P}=$ $0.175)$ and $P P G(r=0.06 ; P=0.58)$ [8]. There was a significant reduction in triglyceride and $\mathrm{C}$-peptide and a significant elevation in adiponectin and HDL-cholesterol (HDL-C). However, a significant difference among three groups was not obtained in any laboratory parameters. Adiponectin is a serum protein secreted by adipocytes and suppresses pro-inflammatory state which is observed in the metabolic syndrome including insulin resistance, hyperglycemia, hypertension, and dyslipidemia 
Table 1. Effects of Low- $(7.5 \mathrm{mg} /$ day), Standard- (15 mg/day) and High-Dose (30 mg/day) Pioglitazone on Efficacy and Safety of Pioglitazone

\begin{tabular}{|c|c|c|c|c|c|c|}
\hline Author & Duration of treatment & & & $7.5 \mathrm{mg}$ & $15 \mathrm{mg}$ & $30 \mathrm{mg}$ \\
\hline \multirow[t]{8}{*}{ Rajagopalan et al [8] } & 12 weeks & Efficacy & HbAlc (\%) & $-0.5 * *$ & $-0.6 * *$ & $-0.7 * *$ \\
\hline & & & FPG (mg/dL) & $-30.6^{* *}$ & $-40.3 * *$ & $-41.1 * *$ \\
\hline & & & C-peptide (ng/mL) & $-0.4 * *$ & $-0.4 * *$ & $-0.8 * *$ \\
\hline & & & Adiponectin $(\mu \mathrm{g} / \mathrm{dL})$ & $+10.6 * *$ & $+11.3 * *$ & $+12.1 * *$ \\
\hline & & & HDL-C (mg/dL) & $+3.3 * *$ & $+3.2 * *$ & $+4.2 * *$ \\
\hline & & Safety & Body weight (kg) & +0.2 & $+0.9 * *$ & $+1.9 * *$ \\
\hline & & & $\mathrm{BMI}\left(\mathrm{kg} / \mathrm{m}^{2}\right)$ & +0.1 & $+0.3 * *$ & $+0.8 * *$ \\
\hline & & & Body fat (\%) & +0.1 & $+0.8 * *$ & $+1.2 * *$ \\
\hline \multirow[t]{5}{*}{ Majima et al [9] } & 6 months & Efficacy & HbA1c (\%) & $-0.61 * *$ & $-0.69 * *$ & \\
\hline & & & HDL-C (mg/dL) & $+3.53 * *$ & $+4.55^{* *}$ & \\
\hline & & Safety & Body weight (kg) & +1.14 & +2.79 & \\
\hline & & & Body fat (\%) & +1.97 & +4.75 & \\
\hline & & & Edema & $2 / 54(3.7 \%)$ & $11 / 41(26.8 \%)$ & \\
\hline \multirow[t]{2}{*}{ Panikar et al [10] } & 6 months & Efficacy & HbA1c (\%) & $\downarrow$ & $\downarrow$ & $\downarrow$ \\
\hline & & Safety & Body weight (kg) & $+0.88 * *$ & $+1.62 * *$ & $+2.72 * *$ \\
\hline \multirow[t]{2}{*}{ Adachi et al [7] } & 2 months & Efficacy & HbA1c (\%) & $-0.8^{*}$ & $-0.7 *$ & \\
\hline & & & $\operatorname{ALT}(\mathrm{U} / \mathrm{mL})$ & $-6.8 *$ & -4.5 & \\
\hline
\end{tabular}

ALT: alanine aminotransferase; BMI: body mass index; BNP: B-type natriuretic peptide; FPG: fasting plasma glucose; IRI: immunoreactive insulin; PPG: postprandial plasma glucose. ${ }^{*} \mathrm{P}<0.1$ and ${ }^{* *} \mathrm{P}<0.05$ vs. baseline, respectively.

[11]. Pioglitazone may increase adiponectin concentrations in patients with type 2 diabetes independently of improvements in blood glucose [11]. The low-dose pioglitazone therapy seems to improve dyslipidemia, insulin resistance and increase adiponectin which is one of the most important therapeutic targets of pioglitazone as well as the standard- and high-dose pioglitazone therapy. Aso et al also reported that the low-dose pioglitazone $(7.5 \mathrm{mg} /$ day) therapy increases serum high molecular weight adiponectin and improves glycemic control in Japanese patients with poorly controlled type 2 diabetes [12]. $\mathrm{Vu}$ et al reported that the low-dose pioglitazone $(7.5 \mathrm{mg} /$ day $)$ therapy increases serum high molecular weight adiponectin and improves glycemic control in patients with the metabolic syndrome without type 2 diabetes [13].

The standard and high dose of pioglitazone showed a significant increase in body weight, body mass index (BMI) and body fat, which was not observed in the low-dose pioglitazone group [8]. The low-dose pioglitazone therapy is more favorable for body weight and body fat as compared with the standard- and high-dose pioglitazone therapy.
Majima et al compared the effects of low-dose pioglitazone $(7.5 \mathrm{mg} /$ day $)$ with those of standard-dose pioglitazone $(15 \mathrm{mg} /$ day $)$ in Japanese women with type 2 diabetes [9]. Both groups showed a significant reduction in $\mathrm{HbAlc}$, FPG, immunoreactive insulin (IRI) and triglyceride, and also showed a significant elevation of HDL-C; however, none of the intergroup differences reached statistical significance [9]. However, in terms of safety, \% change of body weight during the 6-month treatment in the low-dose pioglitazone group was significantly less than that in the standard-dose pioglitazone group $(\mathrm{P}<0.0001)$. The study by Majima et al almost supported the result of the study by Rajagopalan et al.

Twice as many patients reported edema with pioglitazone than with gliclazide in a randomized, double-blind, parallelgroup comparison trial [14]. In the kidney, PPAR $\gamma$ is most abundant in the collecting duct. Thiazolidinediones has been reported to expand body fluid volume through PPAR $\gamma$ stimulation of the epithelial $\mathrm{Na}^{+}$channel-mediated renal salt absorption [15]. Majima et al also showed that the incidence of peripheral edema was significantly much lower in the low-dose 
pioglitazone group $(3.7 \%)$ than in the standard-dose pioglitazone group $(26.8 \%)(\mathrm{P}=0.0014)$. Further, their study showed that the low-dose pioglitazone therapy was more favorable for peripheral edema as compared with the standard-dose pioglitazone therapy.

Panikar et al studied the effect of low $(7.5 \mathrm{mg} /$ day $)$, standard (15 mg/day) and high (30 mg/day) dose of pioglitazone on glycemic control and weight gain in newly diagnosed type 2 diabetes [10]. At the end of 6 months, there was significant weight gain in all three groups from baseline $(\mathrm{P}<0.01)$. Weight gain was greatest in the high-dose pioglitazone group, intermediate in the standard-dose pioglitazone group and least in the low-dose pioglitazone group. The difference was statistically significant between the low-dose pioglitazone group and the high-dose pioglitazone group, and the standard-dose pioglitazone group and the high-dose pioglitazone group, but not between the low-dose pioglitazone group and the standarddose pioglitazone group. There was no significant difference in $\mathrm{HbA} 1 \mathrm{c}$ reduction between the three groups. The dose was significantly correlated with weight gain $(\mathrm{r}=0.254 ; \mathrm{P}<0.001)$, suggesting the dose-dependent effect of pioglitazone on body weight gain.

Pioglitazone has an adverse effect of edema that may result in subsequent heart failure, especially in diabetic patients with coronary artery disease [16-18]. We previously studied the effects of pioglitazone on plasma B-type natriuretic peptide (BNP) which is a sensitive biomarker for diagnosing heart failure [19]. We compared the data before the start of pioglitazone with the data at almost 2 months (mean, 52.6 days) after the start of pioglitazone [7]. Body weight showed a nonsignificant decrease in the daily $7.5 \mathrm{mg}$ pioglitazone-treated group, and showed a non-significant increase in the daily 15 mg pioglitazone-treated group. HbA1c tended to decrease in both groups. Plasma BNP did not change in the daily $7.5 \mathrm{mg}$ pioglitazone-treated group; however, plasma BNP tended to increase in the daily $15 \mathrm{mg}$ pioglitazone-treated group. Our study demonstrated that the daily $7.5 \mathrm{mg}$ pioglitazone-treatment improved $\mathrm{HbAlc}$ without increase of body weight and plasma BNP as compared with the daily $15 \mathrm{mg}$ pioglitazonetreatment [7].

The treatment with thiazolidinediones such as pioglitazone reduces hepatocellular lipid levels by $30-50 \%$ by modulating insulin sensitivity and endocrine function of adipose tissue in type 2 diabetes [20]. The administration of pioglitazone was reported to reduce steatosis and inflammation in liver in patients with non-alcoholic steatohepatitis and type 2 diabetes $[21,22]$. In our study, serum alanine aminotransferase (ALT) tended to decrease in only the daily $7.5 \mathrm{mg}$ pioglitazone-treated group [7].

\section{The Influences of Withdrawal and Daily Dose Reduction of Pioglitazone on Metabolic Param- eters in Patients With Type 2 Diabetes}

To understand the influences of withdrawal or dose reduction of pioglitazone in patients with type 2 diabetes, we retrospectively picked up patients who had undergone withdrawal or daily dose reduction of pioglitazone after a continuous prescription for 3 months or longer, and compared the data before the withdrawal or dose reduction of pioglitazone with the data at 3 or 6 months [23]. In patients who had undergone daily dose reduction, the mean dose of pioglitazone was $29.6 \mathrm{mg}$ at baseline, $11.9 \mathrm{mg}$ at 3 months, and $11.7 \mathrm{mg}$ at 6 months after the dose reduction. The number of subjects who had taken high-dose metformin ( $\geq 1,000 \mathrm{mg}$ ) and dipeptidyl peptidase-4 (DPP-4) inhibitors increased after the withdrawal or dose reduction of pioglitazone in both groups. Although no significant change was observed in plasma glucose and HbA1c levels, body weight significantly decreased at 3 and 6 months after the dose reduction, suggesting the dose-dependent effect of pioglitazone on body weight gain and also supporting the usefulness of the low-dose pioglitazone therapy.

\section{The Dose-Dependent Effect of Pioglitazone on Other Safety-Related Parameters}

A population-based cohort study from France showed that pioglitazone exposure was significantly associated with increased risk of bladder cancer [24]. In this study, a significantly increased risk for high cumulative doses of pioglitazone $(\geq$ $28,000 \mathrm{mg}$, adjusted hazard ratio (HR): 1.75 (95\% confidence interval (CI): 1.22 - 2.50)) and long duration of exposure $(\geq 24$ months, adjusted HR: 1.36 (1.04 - 1.79)) was observed [24]. Although controversial discussions exist about the association between pioglitazone and bladder cancer, the low-dose pioglitazone therapy may be beneficial to reduce the risk of bladder cancer.

In the nested case-control analysis using the UK General Practice Research Database, a possible association between long-term use of thiazolidinediones and fractures was observed in patients with diabetes mellitus [25]. A meta-analysis reported that pioglitazone was associated with a significantly increased risk of fractures overall in the 10 randomized controlled trials (RCTs) (odds ratio (OR): 1.45, 95\% CI: 1.18 1.79 ; $\mathrm{P}<0.001)$ [26]. Five RCTs showed a significantly increased risk of fractures among women (OR: 2.23, 95\% CI: $1.65-3.01 ; \mathrm{P}<0.001)$. Bone mineral density in women exposed to thiazolidinediones was significantly reduced at the lumbar spine and hip in two RCTs [26]. At the moment, the dose-dependent effect of pioglitazone on bone fractures remains unknown.

\section{Conclusion}

The summary about efficacy and safety of the low dose $(7.5 \mathrm{mg} /$ day) pioglitazone therapy was shown in Figure 1. The low-dose pioglitazone therapy may show the same degree of effects on improvements in glucose and lipid metabolism, fatty liver, insulin resistance and adiponectin as the standard- and high-dose pioglitazone therapy. Furthermore, the low-dose pioglitazone therapy may also show less adverse effects on weight gain, edema and heart failure as compared with the standard- and highdose pioglitazone therapy. At this moment, the dose-dependent 


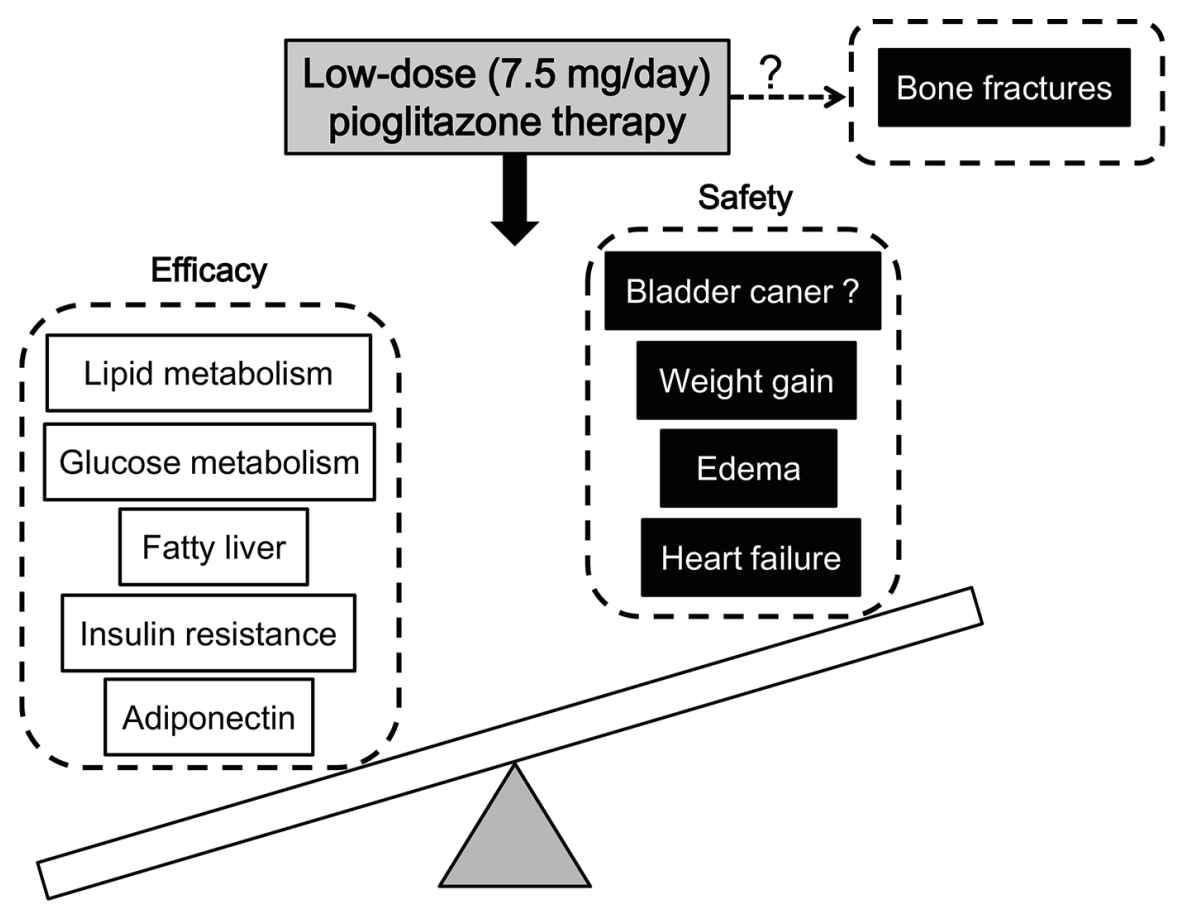

Figure 1. Efficacy and safety of the low-dose $(7.5 \mathrm{mg} /$ day) pioglitazone therapy. Low dose of pioglitazone could improve the efficacy and safety, but reduce the side effect to the minimum, suggesting that low dose might be the optimal dose. Briefly, the low-dose pioglitazone therapy may show the same degree of improvements in glucose and lipid metabolism, fatty liver, insulin resistance, and adiponectin as the standard- and high-dose pioglitazone therapy. Furthermore, the low-dose pioglitazone therapy may also show less adverse effects on weight gain, edema and heart failure as compared with the standard- and high-dose pioglitazone therapy.

effect of pioglitazone on bone fractures remains unknown.

\section{Competing Interests}

The authors declare that they have no competing interests concerning this article.

\section{References}

1. Yki-Jarvinen $\mathrm{H}$. Thiazolidinediones. $\mathrm{N}$ Engl $\mathrm{J}$ Med. 2004;351(11):1106-1118.

2. Kadowaki T, Yamauchi T, Kubota N, Hara K, Ueki K, Tobe K. Adiponectin and adiponectin receptors in insulin resistance, diabetes, and the metabolic syndrome. J Clin Invest. 2006;116(7):1784-1792.

3. Goldberg RB, Kendall DM, Deeg MA, Buse JB, Zagar AJ, Pinaire JA, Tan MH, et al. A comparison of lipid and glycemic effects of pioglitazone and rosiglitazone in patients with type 2 diabetes and dyslipidemia. Diabetes Care. 2005;28(7):1547-1554.

4. Liao HW, Saver JL, Wu YL, Chen TH, Lee M, Ovbiagele B. Pioglitazone and cardiovascular outcomes in patients with insulin resistance, pre-diabetes and type 2 diabetes: a systematic review and meta-analysis. BMJ Open. 2017;7(1):e013927.

5. Lee M, Saver JL, Liao HW, Lin CH, Ovbiagele B. Piogl- itazone for Secondary Stroke Prevention: A Systematic Review and Meta-Analysis. Stroke. 2017;48(2):388-393.

6. Strongman $\mathrm{H}$, Korhonen $\mathrm{P}$, Williams R, Bahmanyar S, Hoti F, Christopher S, Majak M, et al. Pioglitazone and risk of mortality in patients with type 2 diabetes: results from a European multidatabase cohort study. BMJ Open Diabetes Res Care. 2017;5(1):e000364.

7. Adachi H, Katsuyama H, Yanai H. The low dose $(7.5 \mathrm{mg} /$ day) pioglitazone is beneficial to the improvement in metabolic parameters without weight gain and an increase of risk for heart failure. Int J Cardiol. 2017;227:247-248.

8. Rajagopalan S, Dutta P, Hota D, Bhansali A, Srinivasan A, Chakrabarti A. Effect of low dose pioglitazone on glycemic control and insulin resistance in Type 2 diabetes: A randomized, double blind, clinical trial. Diabetes Res Clin Pract. 2015;109(3):e32-35.

9. Majima T, Komatsu Y, Doi K, Shigemoto M, Takagi C, Fukao A, Corners J, et al. Safety and efficacy of low-dose pioglitazone $(7.5 \mathrm{mg} /$ day $)$ vs. standard-dose pioglitazone $(15 \mathrm{mg} /$ day) in Japanese women with type 2 diabetes mellitus. Endocr J. 2006;53(3):325-330.

10. Panikar V, Kale NJ, Hoskote SS, Deogaonkar N, Joshi SR. Effect of Low $(7.5 \mathrm{mg} /$ day $)$, Standard $(15 \mathrm{mg} /$ day $)$ and High $(30 \mathrm{mg} /$ day $)$ Dose Pioglitazone Therapy on Glycemic Control and Weight Gain in Recently-Diagnosed Type 2 Diabetes Patients. J Assoc Physicians India. 2015;63(11):36-39.

11. Schondorf T, Maiworm A, Emmison N, Forst T, Pfutzner 
A. Biological background and role of adiponectin as marker for insulin resistance and cardiovascular risk. Clin Lab. 2005;51(9-10):489-494.

12. Aso Y, Hara K, Ozeki N, Yatsuka C, Nakano T, Matsumoto S, Suetsugu M, et al. Low-dose pioglitazone increases serum high molecular weight adiponectin and improves glycemic control in Japanese patients with poorly controlled type 2 diabetes. Diabetes Res Clin Pract. 2009;85(2):147-152.

13. Vu A, Kosmiski LA, Beitelshees AL, Prigeon R, Sidhom MS, Bredbeck B, Predhomme J, et al. Pharmacodynamic Effects of Low-Dose Pioglitazone in Patients with the Metabolic Syndrome without Diabetes Mellitus. Pharmacotherapy. 2016;36(3):252-262.

14. Charbonnel BH, Matthews DR, Schernthaner G, Hanefeld M, Brunetti P, Group QS. A long-term comparison of pioglitazone and gliclazide in patients with Type 2 diabetes mellitus: a randomized, double-blind, parallel-group comparison trial. Diabet Med. 2005;22(4):399-405.

15. Guan Y, Hao C, Cha DR, Rao R, Lu W, Kohan DE, Magnuson MA, et al. Thiazolidinediones expand body fluid volume through PPARgamma stimulation of ENaC-mediated renal salt absorption. Nat Med. 2005;11(8):861866.

16. Karter AJ, Ahmed AT, Liu J, Moffet HH, Parker MM. Pioglitazone initiation and subsequent hospitalization for congestive heart failure. Diabet Med. 2005;22(8):986993.

17. Ogawa S, Takeuchi K, Ito S. Plasma BNP levels in the treatment of type 2 diabetes with pioglitazone. J Clin Endocrinol Metab. 2003;88(8):3993-3996.

18. Sambanis C, Tziomalos K, Kountana E, Kakavas N, Zografou I, Balaska A, Koulas G, et al. Effect of pioglitazone on heart function and $\mathrm{N}$-terminal pro-brain natriu- retic peptide levels of patients with type 2 diabetes. Acta Diabetol. 2008;45(1):23-30.

19. Calzetta L, Orlandi A, Page C, Rogliani P, Rinaldi B, Rosano G, Cazzola M, et al. Brain natriuretic peptide: Much more than a biomarker. Int J Cardiol. 2016;221:10311038.

20. Roden M. Mechanisms of Disease: hepatic steatosis in type 2 diabetes--pathogenesis and clinical relevance. Nat Clin Pract Endocrinol Metab. 2006;2(6):335-348.

21. Belfort R, Harrison SA, Brown K, Darland C, Finch J, Hardies J, Balas B, et al. A placebo-controlled trial of pioglitazone in subjects with nonalcoholic steatohepatitis. N Engl J Med. 2006;355(22):2297-2307.

22. Teranishi T, Ohara T, Maeda K, Zenibayashi M, Kouyama K, Hirota Y, Kawamitsu H, et al. Effects of pioglitazone and metformin on intracellular lipid content in liver and skeletal muscle of individuals with type 2 diabetes mellitus. Metabolism. 2007;56(10):1418-1424.

23. Katsuyama H, Fukunaga T, Hamasaki H, Adachi H, Moriyama S, Kawaguchi A, Mishima S, et al. The Influences of Withdrawal and Daily Dose Reduction of Pioglitazone on Metabolic Parameters in Patients With Type 2 Diabetes: A Retrospective Longitudinal Observational Study. J Clin Med Res. 2016;8(8):585-590.

24. Neumann A, Weill A, Ricordeau P, Fagot JP, Alla F, Allemand H. Pioglitazone and risk of bladder cancer among diabetic patients in France: a population-based cohort study. Diabetologia. 2012;55(7):1953-1962.

25. Meier C, Kraenzlin ME, Bodmer M, Jick SS, Jick H, Meier CR. Use of thiazolidinediones and fracture risk. Arch Intern Med. 2008;168(8):820-825.

26. Loke YK, Singh S, Furberg CD. Long-term use of thiazolidinediones and fractures in type 2 diabetes: a metaanalysis. CMAJ. 2009;180(1):32-39. 